\title{
گुs \\ Multiharmonic rf feedforward system for compensation of beam loading and periodic transient effects in magnetic-alloy cavities of a proton synchrotron
}

\author{
Fumihiko Tamura, ${ }^{*}$ Chihiro Ohmori, Masanobu Yamamoto, Masahito Yoshii, Alexander Schnase, Masahiro Nomura, \\ Makoto Toda, Taihei Shimada, Katsushi Hasegawa, and Keigo Hara \\ J-PARC Center, KEK \& JAEA, 2-4 Shirakata-Shirane, Tokai, Naka, Ibaraki, Japan 319-1195
}

(Received 27 February 2013; published 20 May 2013)

\begin{abstract}
Beam loading compensation is a key for acceleration of a high intensity proton beam in the main ring (MR) of the Japan Proton Accelerator Research Complex (J-PARC). Magnetic alloy loaded rf cavities with a $Q$ value of 22 are used to achieve high accelerating voltages without a tuning bias loop. The cavity is driven by a single harmonic $(h=9)$ rf signal while the cavity frequency response also covers the neighbor harmonics $(h=8,10)$. Therefore the wake voltage induced by the high intensity beam consists of the three harmonics, $h=8,9,10$. The beam loading of neighbor harmonics is the source of periodic transient effects and a possible source of coupled bunch instabilities. In the article, we analyze the wake voltage induced by the high intensity beam. We employ the rf feedforward method to compensate the beam loading of these three harmonics $(h=8,9,10)$. The full-digital multiharmonic feedforward system was developed for the MR. We describe the system architecture and the commissioning methodology of the feedforward patterns. The commissioning of the feedforward system has been performed by using high intensity beams with $1.0 \times 10^{14}$ proteins per pulse. The impedance seen by the beam is successfully reduced and the longitudinal oscillations due to the beam loading are reduced. By the beam loading compensation, stable high power beam operation is achieved. We also report the reduction of the momentum loss during the debunching process for the slow extraction by the feedforward.
\end{abstract}

DOI: 10.1103/PhysRevSTAB.16.051002

PACS numbers: 29.20. - c, 29.27.- a

\section{INTRODUCTION}

The Japan Proton Accelerator Research Complex (J-PARC) is a high intensity proton accelerator facility, which consists of the $181 \mathrm{MeV}$ linac, the $3 \mathrm{GeV}$ rapid cycling synchrotron (RCS), and the $30 \mathrm{GeV}$ main ring (MR) [1,2]. The MR delivers the beams to the neutrino experiment and the hadron hall by fast extraction (FX) and slow extraction (SX) schemes, respectively.

The parameters of the J-PARC MR and its rf system are listed in Table I. Since the lattice of the MR has an imaginary $\gamma_{t}$, no transition crossing, which causes unstable longitudinal motion of bunches and beam losses, occurs during the acceleration to the top energy. After a ninemonth beam shutdown during the recovery from the Great Tohoku Earthquake, the J-PARC facility resumed beam operation in December 2011. In June 2012, the MR provided $1.0 \times 10^{14}$ protons per pulse (ppp) to the neutrino experiment [3], which corresponds to the beam power of $200 \mathrm{~kW}$ at the repetition period of $2.56 \mathrm{~s}$. A further beam power upgrade is to be done by increasing the beam intensity and by shortening the repetition period.

\footnotetext{
*fumihiko.tamura@j-parc.jp
}

Published by the American Physical Society under the terms of the Creative Commons Attribution 3.0 License. Further distribution of this work must maintain attribution to the author $(s)$ and the published article's title, journal citation, and DOI.
In both RCS and MR, the magnetic-alloy (MA) cavities are employed to realize the high accelerating voltage, which is necessary for acceleration of high intensity proton beams. In case of the MR, the maximum rf voltage is $280 \mathrm{kV}$ with eight cavities [4].

The cavities of the MR are driven by single harmonic $(h=9)$ rf signals, while the cavities in the RCS are driven by dual-harmonic rf signals [5]. The Q value of the MR cavity is set to 22 . The gap impedance calculated as a parallel LCR circuit is plotted in Fig. 1. The frequency response covers the frequency sweep of the accelerating harmonic $(h=9)$, from 1.67 to $1.72 \mathrm{MHz}$, to follow the

TABLE I. Parameters of the J-PARC MR and its rf system.

\begin{tabular}{lc}
\hline \hline Circumference & $1567.5 \mathrm{~m}$ \\
Energy & $3-30 \mathrm{GeV}$ \\
$\gamma_{t}$ & $31.6 i$ \\
Beam intensity & (achieved) $1.0 \times 10^{14} \mathrm{ppp}$ \\
Repetition period & $(\mathrm{FX}) 2.56 \mathrm{~s}$ \\
& $(\mathrm{SX}) 6.00 \mathrm{~s}$ \\
Accelerating frequency & $1.67-1.72 \mathrm{MHz}$ \\
Harmonic number & 9 \\
Number of bunches & 8 \\
Maximum rf voltage & $280 \mathrm{kV}$ \\
No. of cavities & 8 \\
Accelerating gaps in a cavity & 3 \\
Cavity resonant frequency & $1.72 \mathrm{MHz}$ \\
Q value of rf cavity & 22 \\
\hline \hline
\end{tabular}




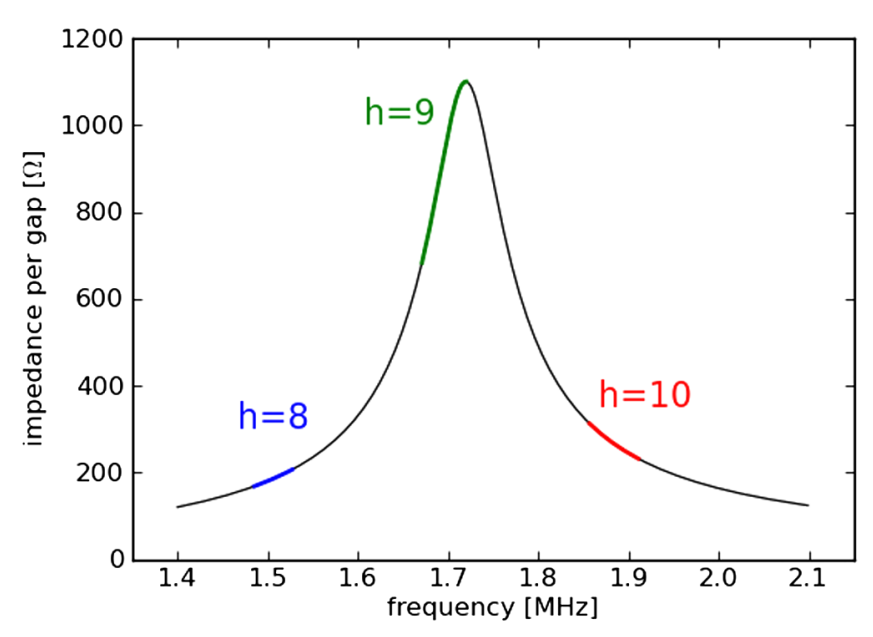

FIG. 1. The frequency response of the accelerating gap and the harmonics covered by the impedance.

velocity change during acceleration without a tuning bias loop, which is usually necessary in case of ferrite cavities. One can notice that the neighbor harmonics $(h=8,10)$ are also covered by the frequency response. The wake voltage in the cavity consists of not only the accelerating harmonic $(h=9)$, but also the neighbor harmonics $(h=8,10)$. The beam loading of the accelerating harmonic $(h=9)$ has effects on the motion of the bunch [6], and that of the neighbor harmonics $(h=8,10)$ are the source of periodic transient effects [7] and they are a possible source of coupled bunch instabilities. Therefore, a multiharmonic beam loading compensation is important for acceleration of high intensity beams. The wake voltage and the beam loading of the high intensity beam in the MA cavity during the injection period are analyzed in Sec. II.

There are several methods to compensate beam loading effects [8]. The direct rf feedback method $[9,10]$ is widely used. The long delay feedback using comb filters can reduce the transient effects. For applying the direct rf

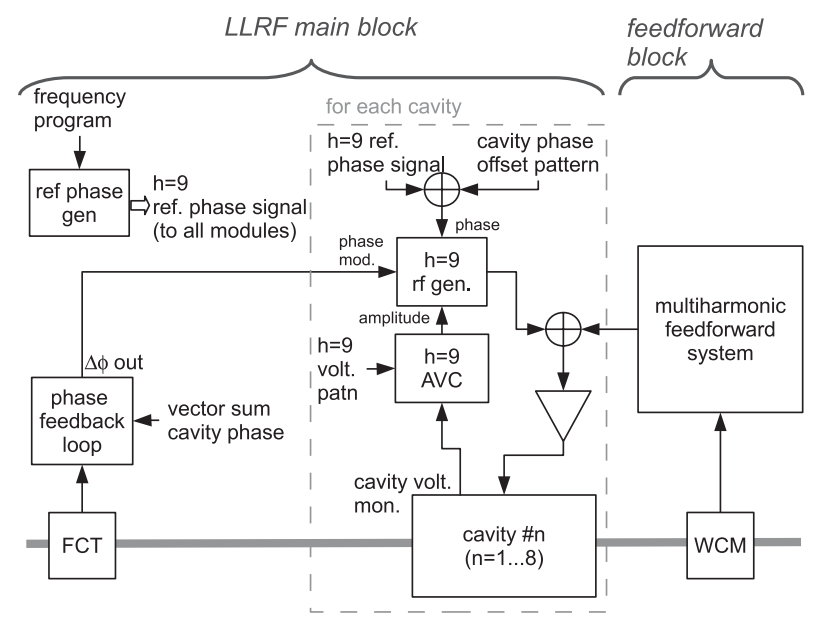

FIG. 2. A simplified block diagram of the MR LLRF control system. feedback method to the J-PARC MR, the feedback amplifier must be located near the cavity in the tunnel to minimize the electrical delay, which determines the feedback gain [9]. There is a space constraint of the MR tunnel, and maintenance of the small feedback amplifier, which may be relatively frequent if it is implemented by using small vacuum tubes, is an issue.

We employ the rf feedforward method for the multiharmonic beam loading compensation, like in the J-PARC RCS, in which the impedance seen by the beam is greatly reduced by the feedforward [11]. The high power system near the cavity is more simple than the case of the direct rf feedback. The multiharmonic feedforward system is developed as a part of the low level rf (LLRF) control system for the MR. Like the other parts of the LLRF control system, the feedforward system is fully implemented by digital circuits to realize the stability and reproducibility of the system. A simplified block diagram of the MR LLRF control system is illustrated in Fig. 2. The MR LLRF control system has a similar architecture of that of the RCS. It consists of the main block and the feedforward block. Each cavity has its own auto voltage control (AVC) loop. The AVC detects the amplitude of the accelerating harmonic $(h=9)$ by using the I/Q demodulation technique, like the AVC of the RCS [12]. The bandwidth of the amplitude detection is narrow, in the order of $100 \mathrm{kHz}$. Therefore, the AVC does not effect the neighbor harmonics $(h=8,10)$. In other words, the AVC does not compensate the wake voltages of the neighbor harmonics at all. The cavity phase is modulated by the phase feedback loop to damp the longitudinal dipole oscillations. The rf signal from the LLRF main block and the output signal of the feedforward system are superposed by a summation amplifier. The function and the architecture of the feedforward system of MR are similar to that of the RCS. We describe the system in Sec. III.

In contrast to the simplicity of the system, the adjustment of the parameters of the feedforward is delicate and its commissioning with beams [8] is time consuming. We apply the commissioning methodology of the multiharmonic feedforward established for the RCS. We review the methodology briefly, and describe the data processing, which is necessary to apply the methodology to the MR with a long accelerating period.

We report the commissioning results and the effects on the beams.

\section{WAKE VOLTAGE IN MR CAVITIES}

In this section, we analyze the wake voltages induced by high intensity beams during the MR injection period.

During the MR injection period, four RCS beam pulses, which consist of two bunches, are transferred into the MR to accumulate eight bunches. The injection interval is $40 \mathrm{~ms}$. The four injection timings are called " $\mathrm{K} 1$," "K2," "K3," and "K4." By turning off the driving rf 
voltage in one of the cavities, the cavity voltage monitor signal consists only of the wake voltage. Since the beam frequency is in the range of $\mathrm{MHz}$, we can observe the wake voltage directly by using an oscilloscope.

Typical waveforms of the wake voltage just after K1-K4 injections are plotted in Fig. 3. $2.5 \times 10^{13}$ protons are transferred to the MR at each injection timing and finally $1.0 \times 10^{14}$ protons are accumulated at K4 timing. The time range of the plot is the revolutional period, $5.32 \mu \mathrm{s}$. The vertical axis is the gap voltage induced by the beam. The bunches are filled from the front with a spacing of $598 \mathrm{~ns}$.

After K1, only two bunches are circulating. Since the Q value of the cavity is relatively low, $Q=22$, the wake voltage decays with time in the revolutional period. In the case of $\mathrm{K} 2$, the wake voltages induced by the four bunches build up until the middle of the revolutional period and then the wake voltage decays towards the end of the period.

The amplitude modulations become smaller at K3 and K4 timing, where six and eight bunches are accumulated, respectively, because most of the rf buckets out of nine, the harmonic number, are filled.

The harmonic components of the wake voltage are plotted in Fig. 4. The waveform of the cavity gap voltage monitor is captured for $10 \mathrm{~ms}$ after every injection timing,
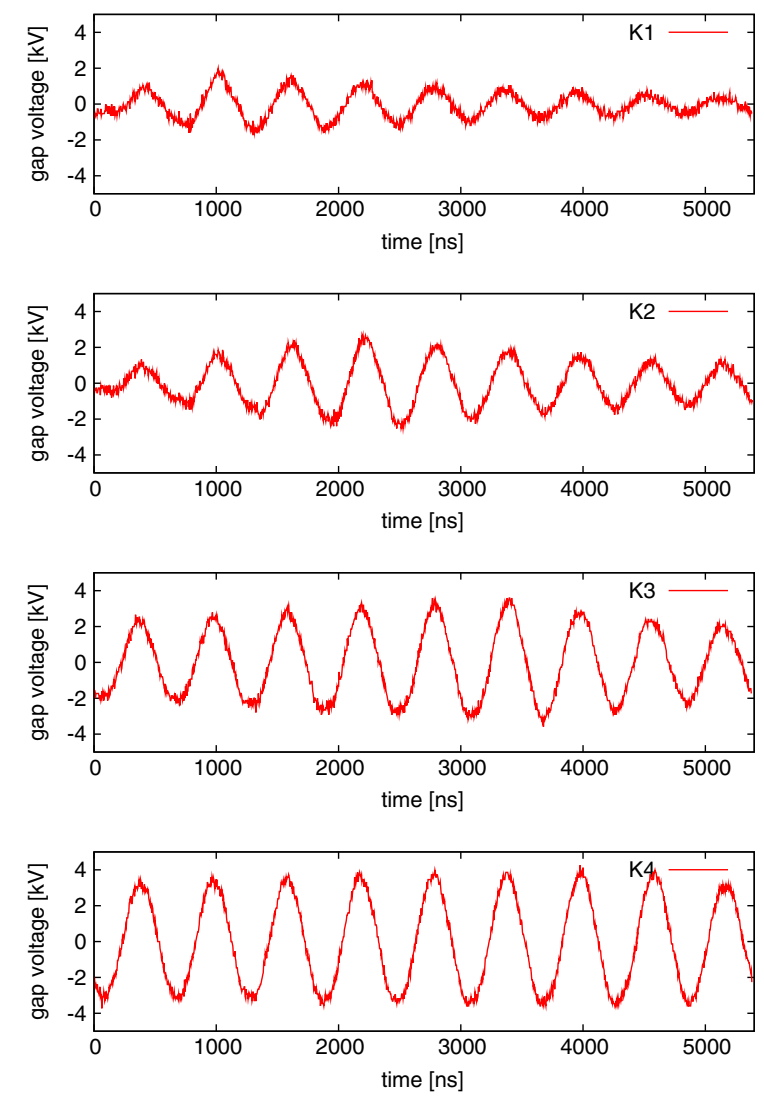

FIG. 3. Typical waveform of the wake voltage just after K1$\mathrm{K} 4$ timings. (Top) K1, (second top) K2, (third top) K3, and (bottom) K4 timing.

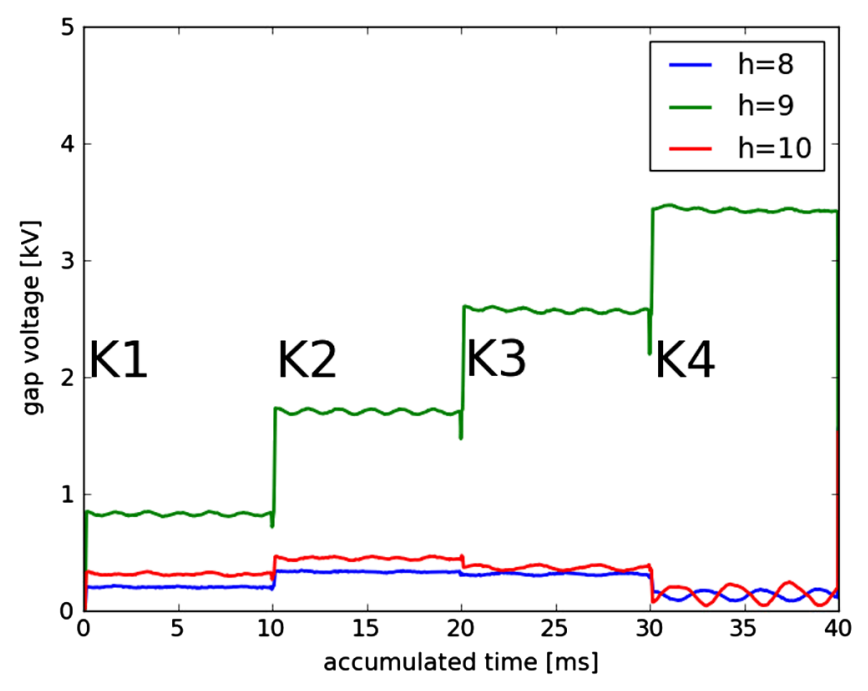

FIG. 4. Harmonic components of the wake voltage.

and the harmonic analysis is performed. Therefore, the horizontal axis in Fig. 4 is the time accumulated in the oscilloscope.

One can see that the amplitude of the accelerating harmonic $(h=9)$ increases proportionally to the number of bunches with filling the rf buckets at the injections, K1 to K4. The components of the neighbor harmonics $(h=8,10)$ correspond to the amplitude modulation during the revolutional period. At $\mathrm{K} 2$, the components of the neighbor harmonics increase. However, since the amplitude modulation of the wake voltage during the revolutional period becomes smaller with filling the rf buckets as described above, the components of the neighbor harmonics decrease at K3 and K4. At K4, the components of the neighbor harmonics $(h=8,10)$ are substantially smaller than that of the accelerating harmonic $(h=9)$.

Hence, the periodic transient effects due to the neighbor harmonics are severe when two and four bunches are injected at $\mathrm{K} 1$ and $\mathrm{K} 2$, respectively. At $\mathrm{K} 4$, the effects become small.

One can notice the oscillations of the amplitude of the components $(h=8,10)$. This is because the phases of the dipole oscillations of the bunches injected from K1 to K4 are different.

When $1.0 \times 10^{14}$ protons are accumulated at $\mathrm{K} 4$, the accelerating harmonic $(h=9)$ component of the wake voltage reaches $3.5 \mathrm{kV}$. In normal operation, the rf voltage during the injection period is set in the range from 5 to $6.25 \mathrm{kV}$ per gap. Thus, the wake voltage is already comparably high with this intensity. The relative loading factor $Y[6]$ is defined by

$$
Y=\frac{I_{b}}{I_{0}},
$$

where $I_{b}$ is the beam current and $I_{0}$ is the resistive current into the cavity. The wake voltage $V_{b}$ and cavity gap voltage $V$ are expressed as 


$$
\begin{gathered}
V_{b}=I_{b} R_{\mathrm{sh}} \cos \phi_{z}, \\
V=I_{0} R_{\mathrm{sh}},
\end{gathered}
$$

respectively, where $R_{\mathrm{sh}}$ is the shunt resistance of the cavity and $\phi_{z}$ is the detuning angle. By combining the equations above, $Y$ is rewritten as

$$
Y=\frac{V_{b}}{V \cos \phi_{z}} .
$$

At the injection frequency, the detuning angle of the MR cavity is $\phi_{z}=51 \mathrm{deg}$. Therefore $Y$ is in the range from 0.9 to 1.1 , with the intensity of $1.0 \times 10^{14} \mathrm{ppp}$. This still satisfies the Robinson stability limit,

$$
Y<\frac{2 \cos \phi_{s}}{\sin 2 \phi_{z}}=2.04,
$$

where the synchronous phase is $\phi_{s}=0$ during the injection period and $\phi_{z}=51 \mathrm{deg}$. However, $Y$ will exceed the limit when the beam intensity is doubled. To increase the beam intensity, beam loading compensation is very important.

The loading angle $\phi_{L}$ is defined by a phase angle between the generator current and the cavity voltage. $\phi_{L}$ is expressed as

$$
\tan \phi_{L}=-\frac{\tan \phi_{z}-Y \cos \phi_{s}}{1+Y \sin \phi_{s}},
$$

and in case of the injection period where $\phi_{s}=0$,

$$
\tan \phi_{L}=Y-\tan \phi_{z} .
$$

Let us assume that $Y=1.0$ at the intensity of $\mathrm{K} 4$, thus $Y=0,0.25,0.5,0.75,1.0$, for the cases of no beam, K1, $\mathrm{K} 2, \mathrm{~K} 3$, and $\mathrm{K} 4$, respectively. Using $\phi_{z}=51 \mathrm{deg}$, the loading angle $\phi_{L}=-51,-44.6,-36.3,-25.9,-13.2 \mathrm{deg}$, varies, respectively. This means that the phase of the cavity voltage relative to the phase reference in the low level rf control (LLRF) system shows a delay due to the beam loading at each injection. The delays are 6.4, 14.7, 25.1, 37.8 deg for K1, K2, K3, and K4, respectively. Since these delays are stepwise at the injection timings, they cause dipole oscillations during the injection period.

Since the auto voltage control of the cavity detects the $(h=9)$ amplitude of cavity voltage to modulate the amplitude of the driving rf voltage from LLRF, the AVC does not work properly if the amplitude of the wake voltage is near the set voltage or exceeds that.

As described above, the beam loading compensation which cancels the wake voltages in the cavity is a key for acceleration of high intensity beams. We employ the rf feedforward method and developed the multiharmonic rf feedforward system to cancel the wake voltages in the accelerating harmonic $(h=9)$ and the neighbor harmonics $(h=8,10)$.

\section{MULTIHARMONIC FEEDFORWARD SYSTEM}

\section{A. Operational principle of $\mathbf{r f}$ feedforward}

The operational principle of the $\mathrm{rf}$ feedforward method is illustrated in Fig. 5. Let us assume the beam current is $i_{\text {beam }}$. The principle is to use the rf amplifier to generate $-i_{\text {beam }}$ in the cavity by using the wall current monitor (WCM) beam signal in addition to the driving rf current for the rf voltage generation, so that the wake voltage is canceled and the impedance seen by the beam approaches zero.

As described in the previous section, the wake voltage consists not only of the accelerating harmonic but also the neighbor harmonics. We developed the multiharmonic feedforward system, which can compensate the beam loading of the most important three harmonics $(h=8,9,10)$.

\section{B. Function of the feedforward system}

We developed the full-digital feedforward system with a similar architecture to that of the RCS [11]. The system compensates the beam loading of the important three harmonics $(h=8,9,10)$.

The block diagram of the feedforward system is shown in Fig. 6. The system uses the WCM signal to generate the feedforward compensation signal, so that the wake voltage can be canceled.

The WCM beam signal is digitized by an analog-todigital converter. The I/Q detection technique is used to pick up the I/Q vectors of the beam signal for the selected harmonics $(h=8,9,10)$. The I/Q vectors of the selected harmonics, $\left(I_{\text {out }}, Q_{\text {out }}\right)$, are obtained as

$$
\begin{aligned}
I_{\text {out }} & =A(h, t) \sin [\phi(h, t)], \\
Q_{\text {out }} & =A(h, t) \cos [\phi(h, t)],
\end{aligned}
$$

where $h$ is the selected harmonics, $t$ the time, $A(h, t)$ and $\phi(h, t)$ are the amplitude and the phase of the selected harmonics, respectively. The I/Q detection blocks use phase reference signals generated by the direct digital synthesis (DDS) technology. Since the driving rf signal is generated by the DDS, the frequency sweep is perfectly synchronized to the driving rf frequency sweep. The I/Q

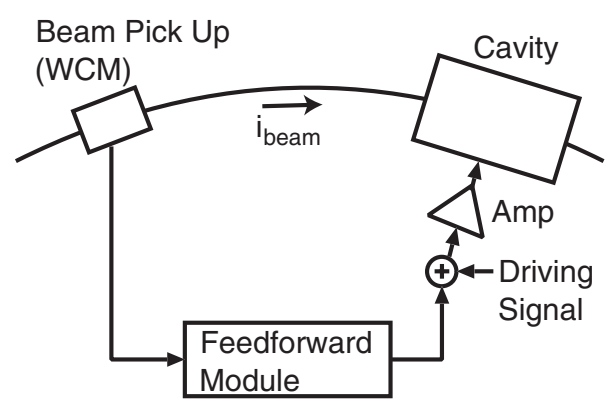

FIG. 5. A conceptual diagram of the rf feedforward method. The feedforward module generates $-i_{\text {beam }}$ so that the wake voltages are canceled. 


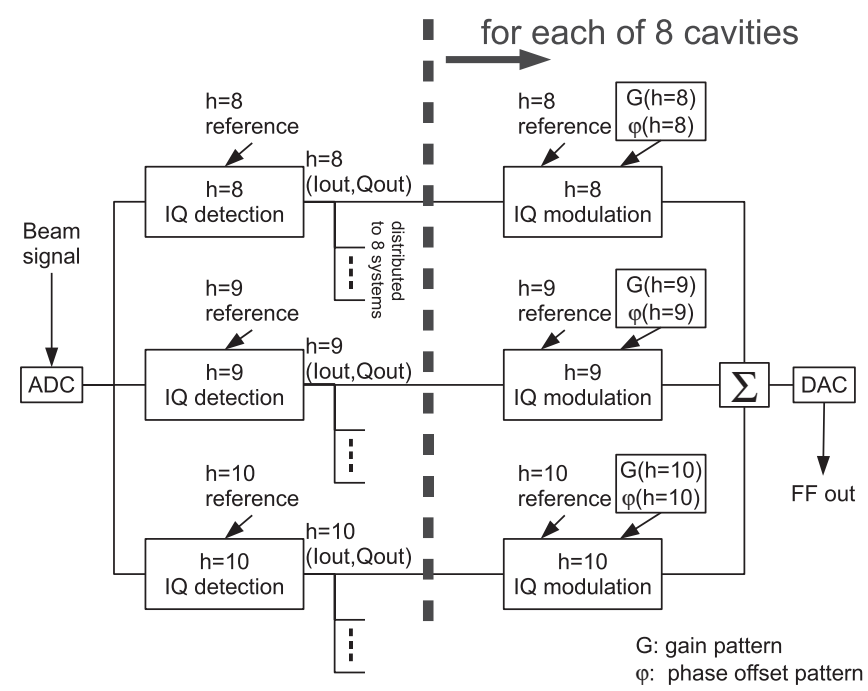

FIG. 6. Block diagram of the multiharmonic rf feedforward system for the MR.

vectors of the harmonics are distributed to the modules for eight cavities.

By using I/Q modulation, feedforward compensation rf signals is generated with gain and phase patterns for the three harmonics. The I/Q modulation output is obtained as

$$
\begin{aligned}
(\mathrm{I} / \mathrm{Q} \text { modulation output })= & G\left[I_{\mathrm{out}} \cos \left(\omega_{h} t+\psi\right)\right. \\
& \left.+Q_{\mathrm{out}} \sin \left(\omega_{h} t+\psi\right)\right] \\
= & G A(h, t) \sin \left[\omega_{h} t+\phi(h, t)+\psi\right],
\end{aligned}
$$

where $G$ and $\psi$ are the gain and phase patterns and $\omega_{h}$ is the angular frequency of the selected harmonic, $\omega_{h}=$ $h \times \omega_{\text {rev }}$ (revolution frequency). The phase references of the harmonics are also used. The compensation signals for the harmonics are summed up and the signal is converted by a digital-to-analog converter. Finally, the compensation signal is sent to the summation amplifier, so that the cavity is driven by a superposition of the driving $\mathrm{rf}$ and the feedforward signal.

The feedforward system works as a tracking band-pass filter, whose passbands at the harmonics $(h=8,9,10)$ follow the frequency sweep with programmed gain and phase patterns. In the feedforward system of the MR, the sampling rate of the feedforward gain and phase patterns is $5 \mathrm{kHz}$. The gain and phase of each passband for $h=8,9,10$ can be set independently in every $0.2 \mathrm{~ms}$, so that the system can follow the change of the optimum parameters from injection to extraction.

\section{COMMISSIONING OF THE FEEDFORWARD SYSTEM}

\section{A. Commissioning methodology}

The commissioning of the feedforward system is to adjust the amplitude and phase patterns for the selected harmonics so that the wake voltages are canceled by the feedforward signals.

In contrast to the commissioning of the digital feedback system $[13,14]$, which is performed without beams to find the optimum parameters and to obtain the maximum stability margins, beam tests are necessary for the commissioning of the feedforward.

The commissioning methodology which is used for the J-PARC RCS is applied to the MR. The details of the methodology is described in [11]. A brief description of the methodology is given below.

Since the frequency sweep of the MR is slow compared to the frequency response of the MA cavity $(Q=22)$, a steady-state equation in frequency domain is a good approximation to calculate the time variation of the cavity gap impedance seen by the beam,

$$
V_{\text {cav }}\left[\omega_{h}(t)\right]=Z_{\text {cav }}\left[\omega_{h}(t)\right] \cdot I_{\text {beam }}\left[\omega_{h}(t)\right],
$$

where $\omega_{h}(t)$ is the angular frequency of the harmonic ( $h=8,9,10), t$ the time, $Z_{\text {cav }}\left[\omega_{h}(t)\right]$ the cavity impedance, $V_{\text {cav }}\left[\omega_{h}(t)\right]$ the complex amplitude for the selected harmonic of the cavity voltage, and $I_{\text {beam }}\left[\omega_{h}(t)\right]$ the complex amplitude of the beam current. The frequencies of the harmonics are obtained as functions of time as programmed. To simplify the notation, we define

$$
\begin{aligned}
V_{\text {cav }}\left[\omega_{h}(t)\right] & =V_{\text {cav }}(h, t), \\
I_{\text {beam }}\left[\omega_{h}(t)\right] & =I_{\text {beam }}(h, t), \\
Z_{\text {cav }}\left[\omega_{h}(t)\right] & =Z_{\text {cav }}(h, t) .
\end{aligned}
$$

The complex amplitudes for the harmonics $(h=8,9,10)$ at the time $t$ of the cavity voltage and the beam current are obtained by performing the harmonic analysis on the waveforms taken by an oscilloscope. Note that the following analysis is in frequency domain.

With feedforward, the accelerating harmonic component $(h=9)$ of the cavity voltage is the superposition of the driving $\mathrm{rf}$ voltage $V_{\text {cav,dr }}(h, t)$, the wake voltage $V_{\text {cav,wake }}(h, t)$, and the feedforward voltage $V_{\text {cav,FF }}(h, t)$, where $h$ is the selected harmonic and $t$ is the time. The superposition is expressed as

$$
\begin{aligned}
V_{\mathrm{cav}}(h, t)= & V_{\mathrm{cav}, \mathrm{dr}}(h, t)+V_{\mathrm{cav}, \text { wake }}(h, t)+V_{\mathrm{cav}, \mathrm{FF}}(h, t) \\
= & H_{\mathrm{dr}}^{\mathrm{cav}}(h, t) \cdot V_{\mathrm{dr}}(h, t)+Z_{\mathrm{cav}}^{\prime}(h, t) \cdot I_{\mathrm{beam}}(h, t) \\
& +Z_{\mathrm{FF}}(h, t) \cdot I_{\text {beam }}(h, t),
\end{aligned}
$$

where $V_{\mathrm{dr}}(h, t)$ and $I_{\text {beam }}(h, t)$ are the harmonic components of the LLRF driving rf signal and the beam current, respectively, $H_{\mathrm{dr}}^{\text {cav }}(h, t)$ the transfer function from the LLRF driving $r$ to the cavity voltage obtained without accelerating a beam, and $Z_{\text {cav }}^{\prime}(h, t)$ the cavity impedance obtained by accelerating a beam without feedforward. By (13), $Z_{\mathrm{FF}}(h, t)$, which is the transfer function from the beam current to the feedforward gap voltage, is obtained by using 
the measured voltage and beam current, $V_{\text {cav }}(h, t)$ and $I_{\text {beam }}(h, t)$. The impedance seen by the beam is

$$
Z_{\mathrm{cav}}^{\prime}(h, t)+Z_{\mathrm{FF}}(h, t) \text {. }
$$

Assuming the linear response of the feedforward voltage, the gain and phase patterns are modified so that

$$
Z_{\mathrm{FF}}(h, t)=-Z_{\text {cav }}^{\prime}(h, t) \text {. }
$$

Several iterations are necessary to realize the minimum impedance seen by the beam.

For the neighbor harmonics ( $h=8,10)$, the cavity voltage is a superposition of the wake voltage and the feedforward voltage as

$$
\begin{aligned}
V_{\text {cav }}(h, t) & =V_{\text {cav, wake }}(h, t)+V_{\text {cav }, \mathrm{FF}}(h, t) \\
& =Z_{\text {cav }}^{\prime}(h, t) \cdot I_{\text {beam }}(h, t)+Z_{\mathrm{FF}}(h, t) \cdot I_{\text {beam }}(h, t) .
\end{aligned}
$$

Similar to the case of the accelerating harmonic $(h=9)$, the gain and phase patterns are adjusted so that $Z_{\mathrm{FF}}(h, t)=$ $-Z_{\mathrm{cav}}^{\prime}(h, t)$.

\section{B. Waveform data processing}

The MR has a much longer cycle, $2.56 \mathrm{~s}$, than that of the $\mathrm{RCS}, 40 \mathrm{~ms}$. The duration from injection to extraction is more than $1.5 \mathrm{~s}$. In case of the RCS, it is possible to capture the waveforms of the voltage monitor signals and the WCM signals from injection to extraction at a high sampling rate by a deep memory oscilloscope. However, it is quite difficult in the case of the MR. For example, if the sampling rate is $200 \mathrm{MHz}$, the waveform data size from injection to extraction exceeds $300 \mathrm{M}$ points, which is too much for data transfer to the PC and analyzing in reasonable time.

To reduce the data size, we take waveforms intermittently during the beam acceleration by utilizing the "sequence mode" of an oscilloscope, Lecroy WP715zi. In the sequence mode, multiple trigger events can be stored as segments into the acquisition memory in the oscilloscope.

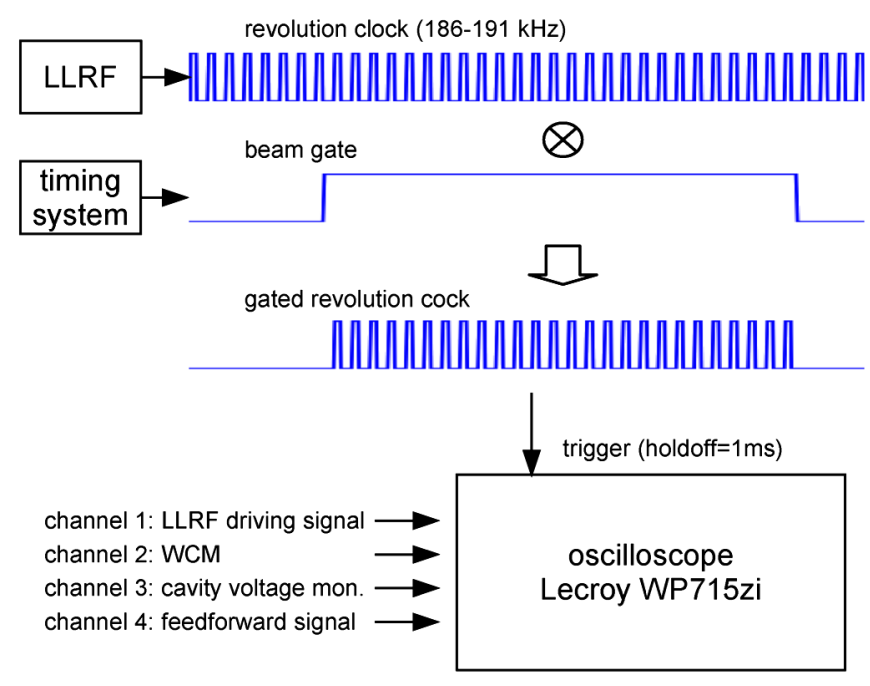

FIG. 7. Signal flow of the segmented waveform capturing.

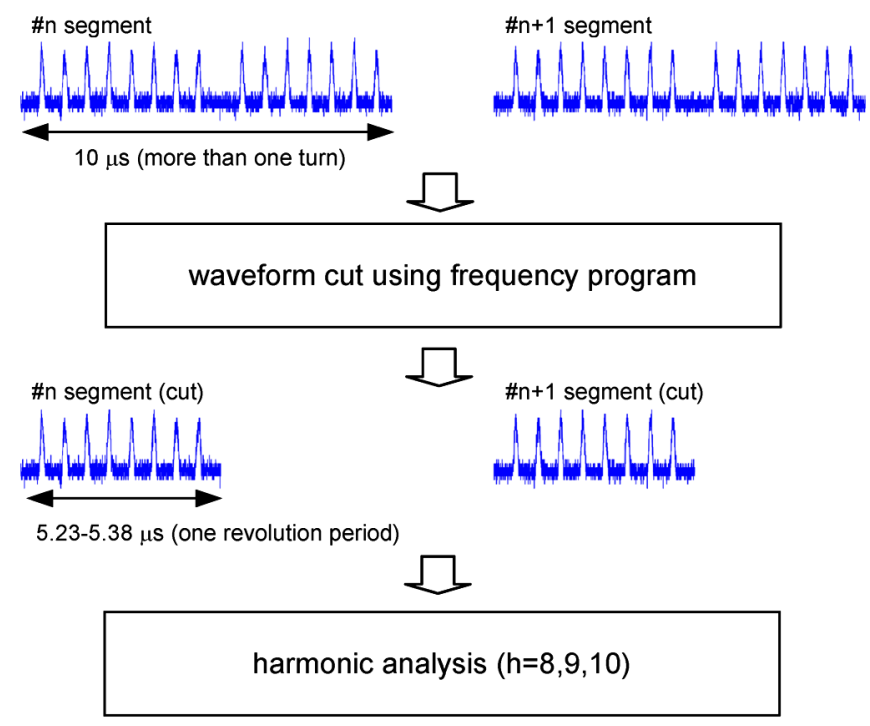

FIG. 8. A schematic of segmented waveform data processing.

A schematic of data taking is illustrated in Fig. 7. A gated revolution clock signal is generated by using the revolution clock from the LLRF control system and the beam gate from the J-PARC timing system. The oscilloscope is triggered by the gated revolution clock signal. The frequency of the revolution clock sweeps from $186 \mathrm{kHz}$ at injection to $191 \mathrm{kHz}$ at extraction. The trigger hold off of the oscilloscope is set to $1 \mathrm{~ms}$, so that the time between the segments is about $1 \mathrm{~ms}$. The sampling rate and the length of each segment are set to $500 \mathrm{MHz}$ and 5000 points, respectively. Hence, one segment length is $10 \mu \mathrm{s}$, which is more than one revolution period, $5.38 \mu \mathrm{s}$ at injection and $5.23 \mu \mathrm{s}$ at extraction. The data size of each channel is in the order of $8 \mathrm{M}$ points and transferring waveforms to the $\mathrm{PC}$ and analysis in the $\mathrm{PC}$ is possible.

The following signals are recorded by the oscilloscope: (i) LLRF driving rf (channel 1); (ii) WCM signal (channel 2); (iii) cavity gap voltage monitor (channel 3); (iv) feedforward output (channel 4).

Each segment, which has $10 \mu$ s length, is shortened to one revolution period by referring to the frequency program to perform the harmonic analysis on the $\mathrm{PC}$, as illustrated in Fig. 8. The harmonic analysis is performed for the accelerating $(h=9)$ and the neighbor harmonics $(h=8,10)$.

\section{Commissioning results}

The feedforward patterns for all eight rf systems were adjusted using a high intensity beam of $1.0 \times 10^{14} \mathrm{ppp}$ by applying the commissioning methodology described above.

A comparison of the impedances and the feedforward transfer function of cavity \#8 is shown in Fig. 9. In the figure, "wake" indicates the impedance seen by the beam without feedforward, "FF component" indicates 

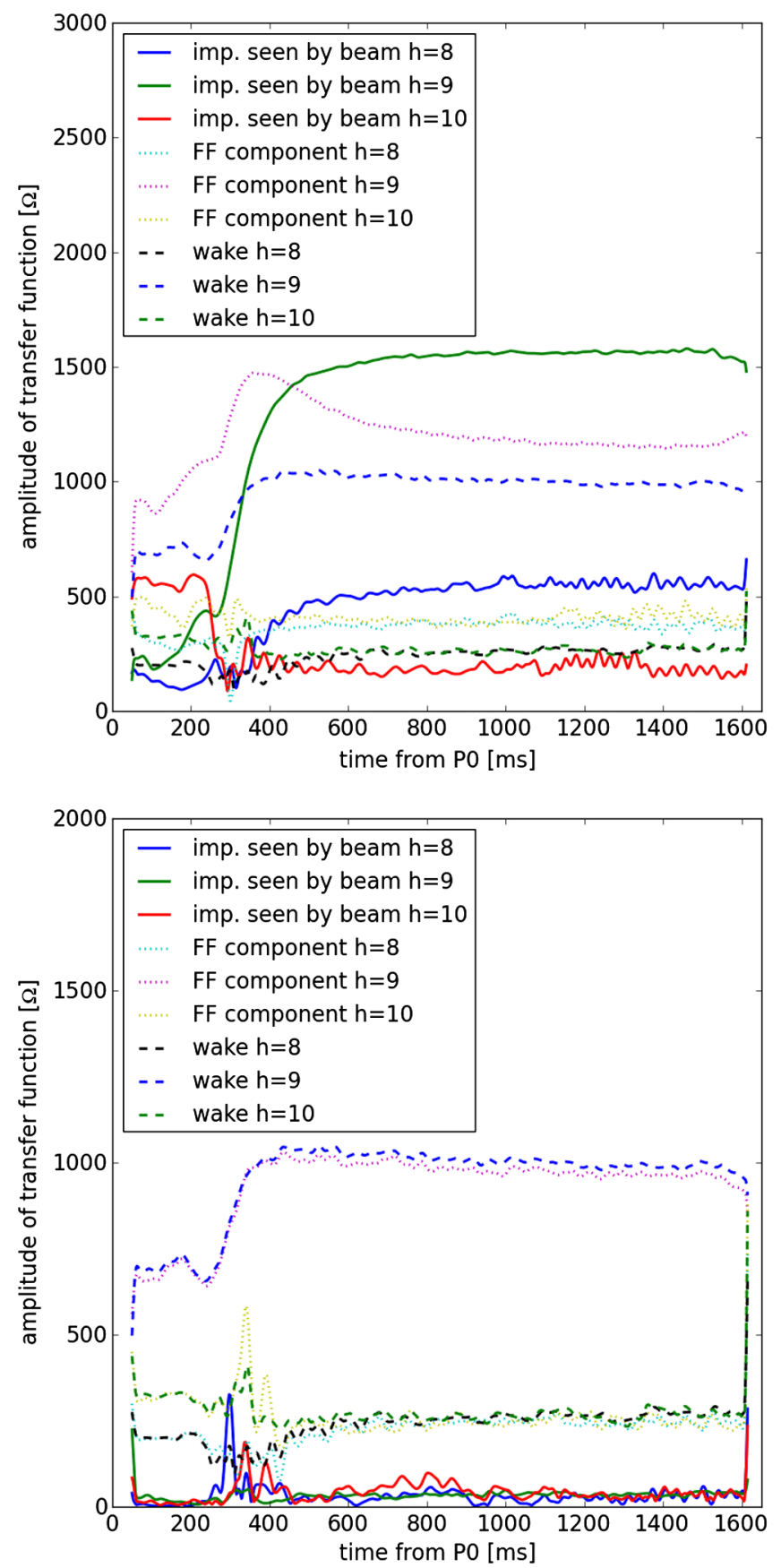

FIG. 9. A comparison of the impedances and the feedforward transfer function of cavity \#8: (top) in case of constant gain and phase patterns for all harmonics; (bottom) the result after two iterations.

the transfer function from the beam current to the feedforward component of the cavity voltage, and "imp. seen by the beam" is the impedance seen by the beam with the feedforward signal. We usually start the commissioning of the feedforward with constant gain and phase patterns for all harmonics. In this case, the amplitude of the feedforward component is far from the impedance seen by the beam without feedforward, as plotted in the top of Fig. 9.
Therefore, the reduction of the impedance seen by the beam is not good and even the increase of the impedance is observed. For example, the impedance seen by the beam with feedforward exceeds the impedance without feedforward after $300 \mathrm{~ms}$. The gain and phase patterns are far from the optimum, however, the cavity voltage does not show any oscillation and is stable, because the feedforward is an open loop.

From this measurement, the gain and phase patterns are modified according to the methodology described in the previous subsection. The comparison after two iterations is plotted at the bottom of Fig. 9. For the accelerating harmonic $(h=9)$, the amplitude of the feedforward component is close to the impedance seen by the beam without feedforward from injection to extraction. Therefore the impedance seen by the beam with feedforward is kept low, less than $50 \Omega$, while the shunt resistance of the single gap is $1100 \Omega$.

For the neighbor harmonics $(h=8,10)$, the errors of the feedforward amplitude remain from 220 to $400 \mathrm{~ms}$ and the reductions of the impedances seen by the beam are not so
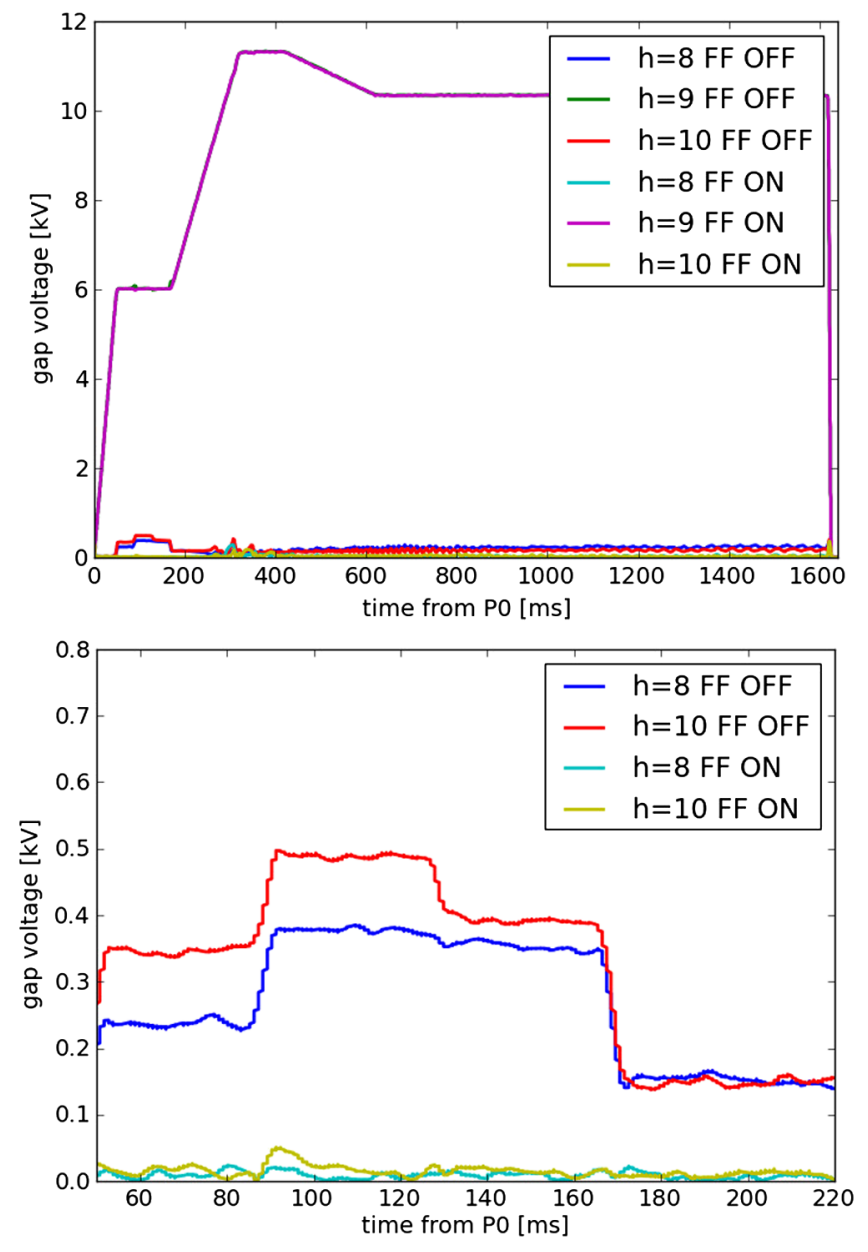

FIG. 10. A comparison of the harmonic components of cavity \#8 gap voltage without and with feedforward; (top) full accelerating cycle; (bottom) magnified plot during injection period. 
good. Other than that, especially during the injection period (until $220 \mathrm{~ms}$ ) where the periodic transient effects are severe, the errors are small and the reductions of the impedance are fairly good.

The harmonic components of cavity \#8 gap voltage, without and with feedforward, are plotted in Fig. 10. The accelerating harmonic components $(h=9)$ are similar without and with feedforward, because the amplitude of the accelerating harmonic component is controlled strongly by the AVC. The reductions of the neighbor harmonics $(h=8,10)$ during injection period are clearly observed, as shown in the bottom plot of Fig. 10. Without feedforward, the harmonic components of the wake voltage in the gap reach 0.37 and $0.5 \mathrm{kV}$, respectively, when four bunches are circulating from 90 to $130 \mathrm{~ms}$. Both components are reduced to less than $0.05 \mathrm{kV}$.

These measurements confirm that the multiharmonic feedforward system works properly.

Transfer functions of the feedforward system \#8 after commissioning measured by a network analyzer are plotted in Fig. 11. In the plots, the thick line shows the transfer function with a combination of all three harmonics. The dashed line shows the transfer function of each harmonics. In the top and bottom of Fig. 11, the transfer functions during injection period and near the extraction are plotted,
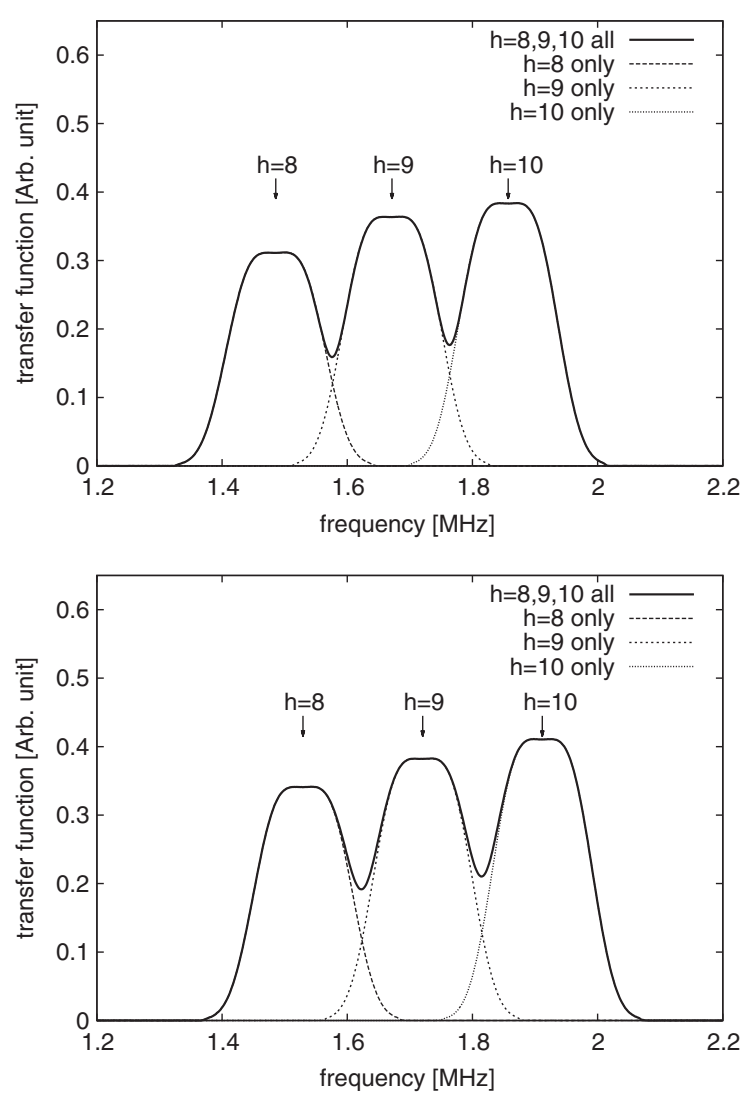

FIG. 11. Transfer functions of the feedforward system \#8 after commissioning: (top) injection period; and (bottom) near extraction. respectively. As described in the previous section, the system works as a tracking band-pass filter. One can see that the passband follows the accelerating frequency sweep from $1.67 \mathrm{MHz}$ at injection to $1.72 \mathrm{MHz}$ at extraction. As the result of the commissioning, the gain is different at injection and extraction. The gain and phase smoothly change according to the gain pattern obtained by the commissioning. The gain around the center of each harmonic is flat in the range of $\Delta f= \pm 20 \mathrm{kHz}$. Since the synchrotron frequency is less than $300 \mathrm{~Hz}$ throughout the acceleration period, the gain flatness around the harmonics is good enough. Looking at the frequency response of each harmonics, one can see that it is zero at the center frequency of the other harmonics. This ensures that one can set the feedforward compensation of each harmonic independently.

The optimum gain and phase patterns are different for each of the cavities, because of the difference of the system characteristics of each system. There are small variations of the resonant frequencies, the shunt resistances, and the $\mathrm{Q}$ values of the cavities. Also, the gains of the tube amplifiers have small variations.

An issue of the commissioning of the feedforward is that it is time consuming. It takes 1 to 1.5 hours to complete the commissioning of each cavity with taking 4 to 6 waveform data. Most of time is used for waveform data processing described in the previous subsection and for the harmonic analysis for three harmonics $(h=8,9,10)$. Since the harmonic analysis performed on the PC is the I/Q demodulation technique similar to the process in the feedforward module, we are considering to develop a hardware, which performs the harmonic analysis of the voltage and current monitor signals. It will greatly reduce the time for commissioning.

\section{EFFECTS OF FEEDFORWARD ON THE BEAM}

The feedforward patterns for all eight rf systems have been commissioned. In this section, we describe the effects of the feedforward on the beam behavior.

\section{A. High power operation}

In this subsection, we describe the beam tests using a high intensity proton beam with $1.0 \times 10^{14} \mathrm{ppp}$. The feedforward patterns for all eight rf systems were commissioned using the high intensity beam. Since the periodic transient effects are severe during the injection period, we apply additional gain patterns for the neighbor harmonics $(h=8,10)$, so that the gains are linearly reduced to zero from the K4 timing to K4 $130 \mathrm{~ms}$. For the accelerating harmonic $(h=9)$, the beam loading is compensated from injection to extraction.

The mountain plots of the WCM beam signal during injection period without and with feedforward are shown in Fig. 12. Similar to Fig. 4, the waveform of the $\mathrm{WCM}$ is captured for $10 \mathrm{~ms}$ after every injection timing. 

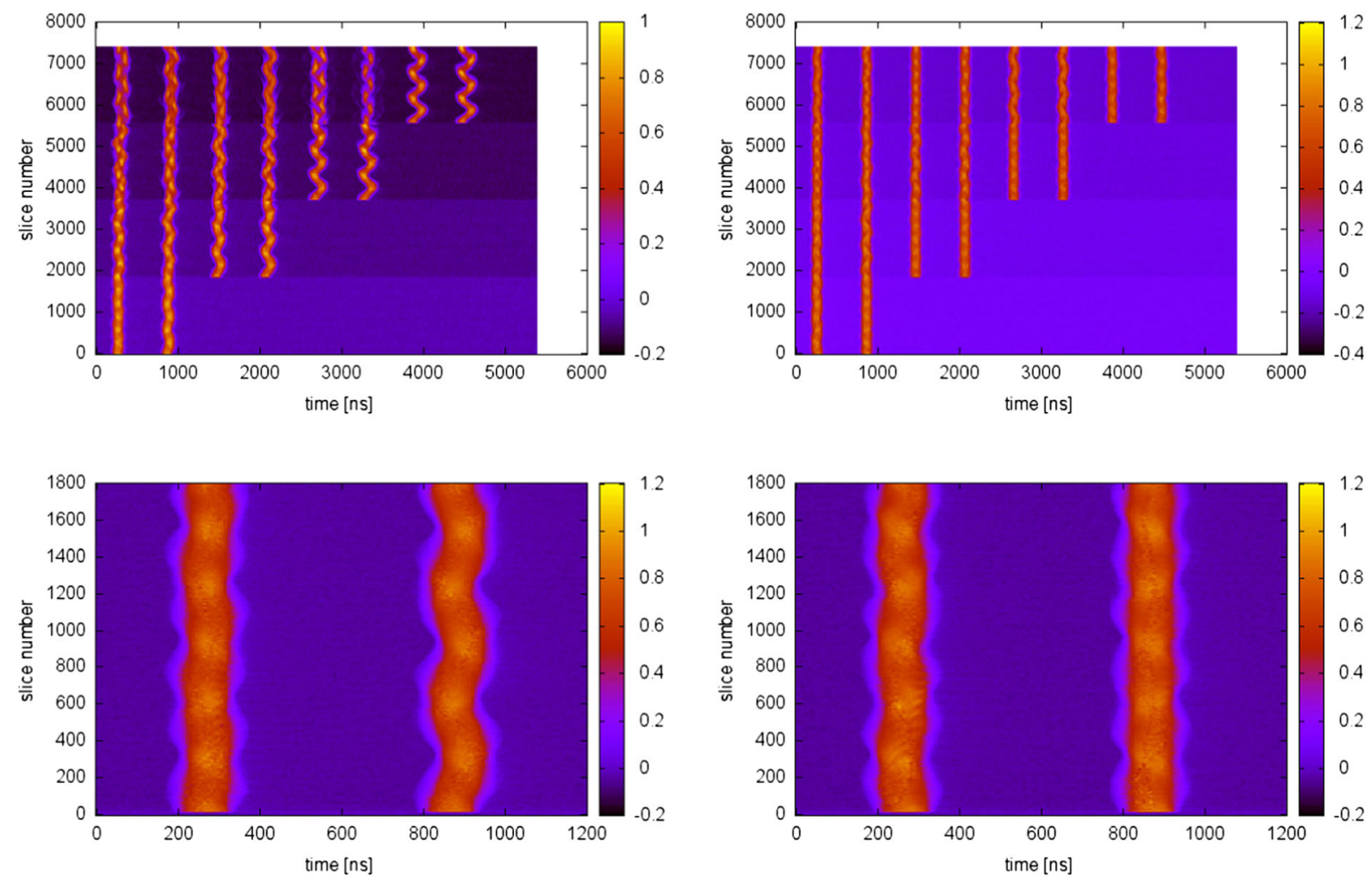

FIG. 12. Mountain plots of the WCM signals during the injection period (left) without and (right) with feedforward. Bottom plots are magnified ones just after $\mathrm{K} 1$ injection timing.

The vertical axis is the slice number, which corresponds to the number of accumulated beam revolutions. In this case, the beam circulates 1857 turns for $10 \mathrm{~ms}$.

As described in Sec. II, the rf phase jumps at every injection timing due to the change of the beam loading angle. The jumps become larger with accumulated beam, and the phase jumps cause longitudinal dipole oscillations. One can notice that dipole oscillations of the injected beams become larger and larger at K2 to K4, without feedforward (upper left of Fig. 12). Also, the circulating bunches are affected by the phase jumps. With feedforward, the beam loading angle is kept almost constant and the rf phase jumps are much reduced. Therefore the dipole oscillations due to the beam loading of the accelerating harmonic are suppressed by feedforward.

The periodic transient effects due to the neighbor harmonics $(h=8,10)$ are severe when two and four bunches are injected at $\mathrm{K} 1$ and $\mathrm{K} 2$, respectively. The lower plots in Fig. 12 are magnified plots of the bunches injected at K1. Without feedforward, the front and rear bunches oscillate differently. The rear bunch shows dipole oscillation, while the front bunch oscillates less. This is because of the periodic transient effects. With the feedforward, the periodic transient effects are suppressed and the behaviors of the front and rear bunches are similar. The amplitude of the oscillation is reduced.
Thus, the multiharmonic feedforward greatly reduces the longitudinal oscillations due to the beam loading and the periodic transient effects during the injection period.

The mountain plots of the WCM beam signal from injection to extraction without and with feedforward are shown in Fig. 13. In this case, the waveform slice of the WCM for one turn is captured every $1.015 \mathrm{~ms}$. Without feedforward (upper left of Fig. 13), the bunches are oscillating throughout the acceleration process. The oscillation starts due to the beam loading during the injection period as described above. Furthermore, the oscillation grows from the beginning of the smoothing period of the bending magnet (slice number 220). The magnified view of the K1 injected bunch during the period is shown in the lower left of Fig. 13. Since each bunch oscillates differently, the phase feedback loop, which detects the average phase of all the bunches, cannot damp the oscillation effectively after the loop is closed at slice number 170 . The average momentum deviations $(d p / p)$ of the beam calculated by using beam position monitor (BPM) signals without and with feedforward are plotted in Fig. 14. A large momentum oscillation in the order of $\pm 0.1 \%$ is observed from 0.3 to $0.8 \mathrm{~s}$ in the upper plot. Because of the momentum oscillation, beam losses in the arc sections with high dispersion are observed as shown in Fig. 15.

The oscillation is much smaller throughout the acceleration period with feedforward as shown in the upper right of 

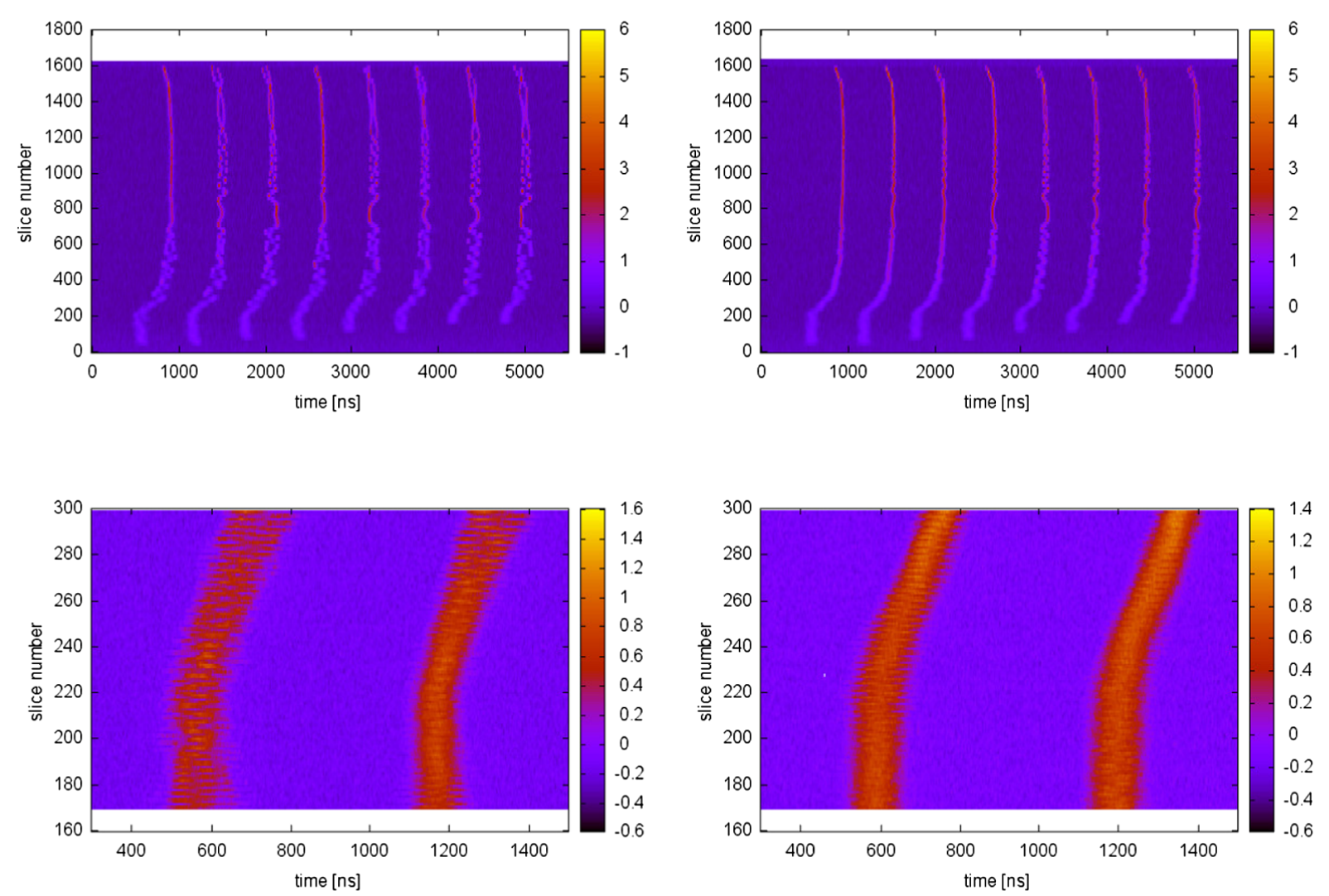

FIG. 13. Mountain plots of the WCM signals from injection to extraction (left) without and (right) with feedforward. Bottom plots are magnified ones of the K1 injected bunches around the smoothing period.

Fig. 12. One can notice that no growth of the oscillation during the smoothing period is observed in the lower right plot. The average momentum deviation becomes much smaller as shown in the lower plot of Fig. 14. Therefore, the beam losses in the arc sections disappear with feedforward, as shown in Fig. 15.

The beam loading compensation by the multiharmonic feedforward is indispensable to realize the high power
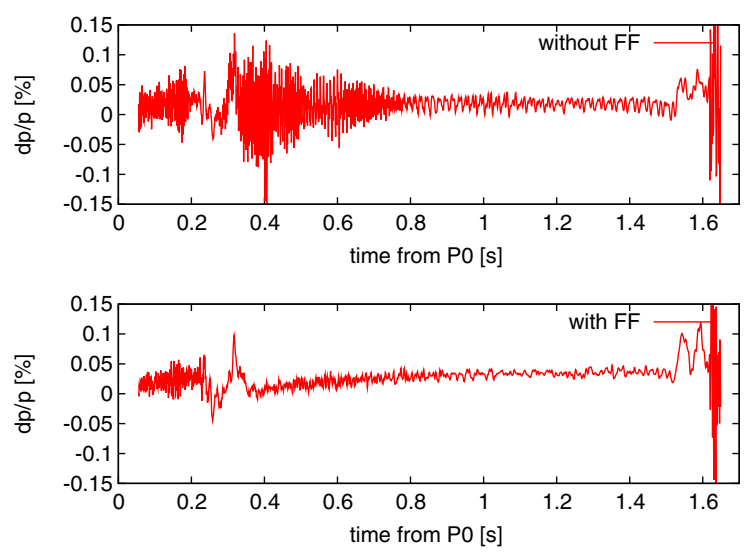

FIG. 14. Typical momentum deviation $(d p / p)$ calculated by using the BPM signals (top) without and (bottom) with feedforward. operation in the J-PARC MR. The feedforward compensation is used in the normal user operation.

\section{B. Reduction of momentum loss during debunching process for slow extraction}

For the hadron experiments, the accelerated beam at $30 \mathrm{GeV}$ is slowly extracted by the third integer resonant extraction $[15,16]$. At present, a very high extraction

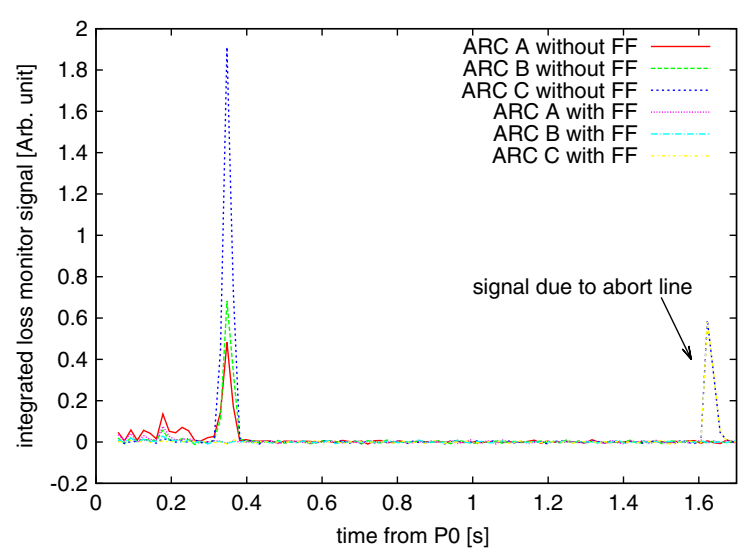

FIG. 15. Typical beam loss monitor signal in the arc sections without and with feedforward. 
efficiency of $99.5 \%$ has been achieved. Such a high efficiency, i.e., a low beam loss is realized by moving the bump orbit dynamically during the extraction process. To increase the beam power, it is very important to keep the high efficiency to avoid damages and activation of the slow extraction devices.

The rf voltage in the cavities is turned off after finishing the acceleration to obtain a coasting beam. During the debunching process over several hundreds of milliseconds, the average momentum decreases due to the longitudinal coupling impedances in the MR. The deceleration continues until a well debunched beam, i.e., a coasting beam is formed. The momentum loss depends on the beam intensity. At the intensity of $7 \times 10^{12} \mathrm{ppp}$, which corresponds to the beam power of $6 \mathrm{~kW}$ at the repetition period of $6 \mathrm{~s}$, the energy loss reaches in the order of $1 \%$. While the chromaticity of the MR is kept zero by two families of the sextupole magnets, the correction is not perfect for a large momentum deviation and the chromatic tune spread affects the extraction separatrix. As a result, the extraction efficiency become worse, about $97 \%$.

The major impedance source is the rf cavity. Possible other sources are the resistive wall impedance and the impedances of the injection and extraction kickers. The impedance of the rf cavities can be reduced by shorting the accelerating gaps in the cavities by mechanical vacuum relays. Also, it is possible to reduce the impedance by the feedforward.

A comparison of the momentum deviation in three cases without the gap short and no feedforward, with gap short, and with feedforward, is shown in Fig. 16. The beam intensity is $7 \times 10^{12} \mathrm{ppp}$.

The momentum deviation is calculated by using the closed orbit taken by beam position monitors and the dispersion function of the ring. The beam position monitor signals become more inaccurate as the debunching proceeds. The horizontal axis is the time from the MR cycle start (P0). The timing at the end of the acceleration is called

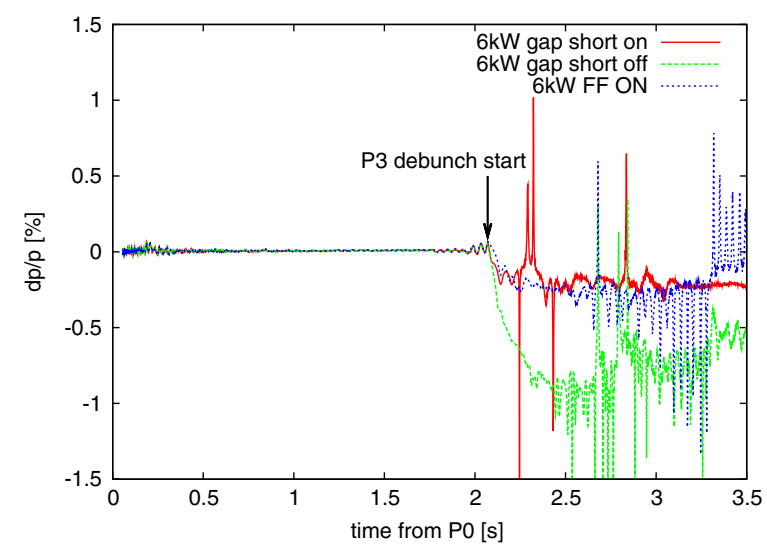

FIG. 16. Comparison of the momentum deviation during the debunching process, without feedforward and gap short, with feedforward, and with gap short.
$\mathrm{P} 3$, it is $2.07 \mathrm{~s}$ from $\mathrm{P} 0$. At $\mathrm{P} 3$, the rf voltage program becomes zero. Without the gap short and no feedforward, the momentum loss during the debunching process was in the order of $1 \%$. The activation signals of the gap short relays are sent right after P3. Since the relay is a mechanical relay, it takes about $30 \mathrm{~ms}$ to close the relay contacts. Until the gap shorts close, an unignorable momentum loss is observed. Despite that, the total momentum loss was reduced down to $0.2 \%$.

The feedforward patterns were adjusted for this operation, because the optimum patterns are different from the high power operation in the FX mode. The wake voltages during debunching were canceled by feedforward and the momentum loss was also reduced to $0.2 \%$ as well as with the gap short.

With either of the gap short and feedforward, the extraction efficiency was kept high, at $99.5 \%$. The operation of the gap short relay has a risk, because the relay can be easily broken if it is closed under the driving rf current. Careful timing control is necessary. In case of the feedforward, we can avoid such kind of risks. Thus, the feedforward has been used for the user operation.

It has been demonstrated that up to the intensity of $1.7 \times 10^{13} \mathrm{ppp}$, which corresponds to the beam power of $14 \mathrm{~kW}$, the extraction efficiency was kept at $99.5 \%$ despite the momentum loss, which increased to $0.3 \%$.

These results are encouraging for achieving future high power operation at more than $50 \mathrm{~kW}$, although it is expected that retuning of the feedforward will be necessary.

\section{SUMMARY AND DISCUSSIONS}

We summarize the article as follows.

1. For the high intensity operation of the J-PARC MR, a multiharmonic beam loading compensation, which cancels the wake voltages of the accelerating harmonic and the neighbor harmonics in the MA cavities, is indispensable.

2. The rf feedforward method is employed for the beam loading compensation in the MR, like in the RCS in which the impedance seen by the beam is greatly reduced by the feedforward.

3. A full-digital multiharmonic feedforward compensation system is developed as a part of LLRF control system. The system has a similar architecture to that of the RCS. The feedforward system can cancel the most important three harmonics $(h=8,9,10)$. The system works as a tracking band-pass filter with the passbands of the harmonics, whose passband follows the accelerating frequency sweep with the programmed gain and phase patterns.

4. The commissioning methodology of the feedforward used for the RCS is applied to the MR. To adapt to the long accelerating period, a data processing technique has been developed. All eight systems have been commissioned. The impedances seen by the beam for the important harmonics $(h=8,9,10)$ are reduced with feedforward. 
5. The longitudinal oscillations due to the beam loading of high intensity beams are reduced by the feedforward. The stable high power operation is possible with feedforward.

6. The momentum loss during the debunching period due to the cavity impedance is a major issue to achieve high extraction efficiency of the slow extraction. The momentum loss is substantially reduced with feedforward.

To achieve higher beam power, it is proposed to employ a repetition rate of $1 \mathrm{~Hz}$, higher than that of $0.4 \mathrm{~Hz}$ at present [17]. To achieve the high repetition rate, a new main magnet power supply and an rf cavity with a higher field gradient are necessary. The R\&D works are ongoing. The higher gradient rf cavity can be realized by using improved MA core material, which has a higher impedance than that currently used in the J-PARC synchrotrons [18]. By employing the higher gradient cavities, an accelerating voltage of more than $500 \mathrm{kV}$ will be generated by nine cavities, while $280 \mathrm{kV}$ is generated by eight cavities at present. However, the beam loading of the higher gradient cavity will be severer than at present due to the higher impedance. Thus, the beam loading compensation by the multiharmonic feedforward will be more important. The commissioning methodology can be applied to the shorter accelerating period.

\section{ACKNOWLEDGMENTS}

We would like to thank Professor E. Ezura, Professor K. Takata, Professor E. Takasaki, and Professor A. Ando for fruitful discussions and comments. We also thank the beam commissioning group of the MR for providing several days of beam time for commissioning of all the feedforward systems. Also, we would like to thank all the members of the J-PARC.

[1] JHF Project Office, KEK Report 97-16, 1997.

[2] High-intensity Proton Accelerator Project Team, JAERITECH 2003-044, 2003.
[3] K. Abe et al. (T2K Collaboration), Phys. Rev. Lett. 107, 041801 (2011).

[4] M. Yoshii et al., in Proceedings of the 3rd International Particle Accelerator Conference, New Orleans, Louisiana, USA, 2012 (IEEE, Piscataway, NJ, 2012), pp. 3281-3283.

[5] F. Tamura et al., Phys. Rev. ST Accel. Beams 12, 041001 (2009).

[6] F. Pedersen, IEEE Trans. Nucl. Sci. 22, 1906 (1975).

[7] M. Yamamoto et al., in Proceedings of the 18th Particle Accelerator Conference, New York, 1999 (IEEE, New York, 1999), pp. 860-862.

[8] R. Garoby, in Proceedings of the U.S.-CERN-Japan International School (World Scientific, Hayama and Tsukuba, 1996), pp. 455-489.

[9] D. Boussard, in Proceedings of the 1985 Particle Accelerator Conference (IEEE, Vancouver, BC, Canada, 1985), pp. 1852-1856 [http://epaper.kek.jp/p85/INDEX .HTM].

[10] D. Boussard, in Proceedings of CERN Accelerator School, Oxford (CERN92-03, 1991), pp. 474-500.

[11] F. Tamura, M. Yamamoto, C. Ohmori, A. Schnase, M. Yoshii, M. Nomura, M. Toda, T. Shimada, K. Hara, and K. Hasegawa, Phys. Rev. ST Accel. Beams 14, 051004 (2011).

[12] F. Tamura, A. Schnase, and M. Yoshii, Phys. Rev. ST Accel. Beams 11, 072001 (2008).

[13] D. Teytelman, in Proceedings of the 21st Particle Accelerator Conference, Knoxville, 2005 (IEEE, Piscataway, NJ, 2005), pp. 2863-2865.

[14] D. V. Winkle et al., in Proceedings of the International Particle Accelerator Conference, Kyoto, Japan (IPAC'10 OC/ACFA, Kyoto, 2010), pp. 1479-1481.

[15] H. G. Hereward, CERN Technical Report No. CERN-ARInt-GS-61-5, 1961.

[16] M. Tomizawa et al., in in Proceedings of the 3rd International Particle Accelerator Conference, New Orleans, Louisiana, USA, 2012 (Ref. [4]), pp. 481-483.

[17] T. Koseki et al., Prog. Theor. Exp. Phys. (2012) [http://ptep.oxfordjournals.org/content/2012/1/02B004 .abstract].

[18] C. Ohmori et al., in Proceedings of the 2nd International Particle Accelerator Conference, San Sebastián, Spain (EPS-AG, Spain, 2011), pp. 2885-2887. 